\title{
Nanoscale
}

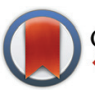

CrossMark \&lick for updates

Cite this: Nanoscale, 2016, 8, 1994

Received 3rd October 2015, Accepted 11th December 2015

DOI: $10.1039 / \mathrm{c} 5 \mathrm{nr} 06855 \mathrm{j}$

www.rsc.org/nanoscale

\section{An excellent candidate for largely reducing interfacial thermal resistance: a nano-confined mass graded interface}

\begin{abstract}
Yanguang Zhou, ${ }^{a}$ Xiaoliang Zhang ${ }^{b}$ and Ming Hu${ }^{\star a, b}$
Pursuing extremely low interfacial thermal resistance has long been the task of many researchers in the area of nano-scale heat transfer, in particular pertaining to improve heat dissipation performance in electronic cooling. While it is well known and documented that confining a macroscopic third layer between two dissimilar materials usually increases the overall interfacial thermal resistance, no research has realized the fundamental decrease in resistance so far. By performing nonequilibrium molecular dynamics simulations, we report that the overall interfacial thermal resistance can be reduced by 6 fold by confining mass graded materials with thickness of the order of nanometers. As comparison we also studied the thermal transport across the perfectly abrupt interface and the widely used alloyed (rough) interface, which shows an opposing and significantly large increase in the overall thermal resistance. With the help of frequency dependent interfacial thermal conductance and wave packet dynamics simulation, different mechanisms governing the heat transfer across these three types of interfaces are identified. It is found that for the rough interface there are two different regimes of interfacial heat transfer, which originates from the competition between phonon scattering and the thickness of the interface. The mechanism of dramatically improved interfacial heat transfer across the nano-confined mass graded interface resides in the minor phonon reflection when the phonons first reach the mass graded area and the rare occurrence of phonon scattering in the subsequent interior region. The phonons are found to be gradually truncated by the geometric interfaces and can travel through the mass graded layer with a high transmission coefficient, benefited from the small mass mismatch between two neighboring layers in the interfacial region. Our findings provide deep insight into the phonon transport across nano-confined mass graded layers and also offer significant guidance for designing advanced thermal interface materials.
\end{abstract}

An interface plays a critical role in determining the overall physical properties of materials, for instance, the thermal and mechanical characters of devices can be modulated when the grain boundaries, ${ }^{1}$ heterojunctions ${ }^{2}$ or aggregates $^{3}$ are involved. Therefore, deep understanding of the physical mechanisms at interfaces is crucial to improve the functions and performance of structures and devices in a diverse spectrum of technologies, such as heat dissipation of electronics.

In the context of phonon dominant thermal transport, interfaces present an additional site for phonon scattering and impediment to the propagation of thermal energy. ${ }^{4}$ The accompanying Kapitza resistance ${ }^{5}$ then results in a number of

${ }^{a}$ Aachen Institute for Advanced Study in Computational Engineering Science (AICES), RWTH Aachen University, 52062 Aachen, Germany.

E-mail: hum@ghi.rwth-aachen.de

${ }^{b}$ Institute of Mineral Engineering, Division of Materials Science and Engineering, Faculty of Georesources and Materials Engineering, RWTH Aachen University,

52064 Aachen, Germany challenges in realizing an as low as possible overall thermal resistance for thermal management of nanoelectronic devices. To address this challenge, engineering the geometry of interfaces such as adding a third layer ${ }^{6,7}$ with nanoscale thickness and incorporating roughness at the interfaces ${ }^{8-11}$ are the two most popular methods. For example, experimental results have shown that thermal boundary resistance increases as the roughness of interfaces increases, which is generated through chemical etching, or as the thickness of the third layer aggrandizes. ${ }^{8,10}$ At the same time, from the theoretical aspect, the interfacial thermal resistance of such structures has been investigated using the modified classical acoustic mismatch model $(\mathrm{AMM}),{ }^{12,13}$ diffuse mismatch model $(\mathrm{DMM})^{14}$ and atomistic Green's function (AGF). ${ }^{15}$ However, all these three approaches have their limitations (the AMM is only valid for long wavelength phonons, the DMM assumes a fully diffusively scattering interface and the AGF models the phonons in the ballistic transport regime) and then lead to the inaccuracy of the predicted thermal resistance. Most recently, using classical molecular 
dynamics (MD) simulations, such sandwiched interfacial structures were studied and the thermal resistance was found to be related to the thickness and the detailed atomic configuration of the interlayer. ${ }^{12,16-23}$ However, almost all the results mentioned above show that the overall interfacial thermal resistance tends to increase, when a third layer is involved. Only with an extremely thin interlayer, which is significantly difficult to fabricate, the thermal resistance of such structures can be reduced by about $20 \%$ to $50 \%$ at the most. ${ }^{15,16,18,20}$ In addition, how the phonons interact in the interfacial region for the sandwiched structures is still unclear. Therefore, designing a structure which can significantly reduce the overall interfacial thermal resistance and understanding the detailed mechanism of phonon transport in the interfacial region are among the few remaining challenges in the interfacial heat transfer community.

In this paper, we uncover an excellent candidate, namely a mass graded interface (MGI) to dramatically decrease the interfacial thermal resistance (ITR), which has already been shown to effectively tune the interfacial electronic properties. ${ }^{24} \mathrm{We}$ found that the mass graded structure can easily reduce the overall ITR by 6 fold with respect to the perfectly abrupt interface (we call it "PI" hereafter). At the same time, the commonly used rough (alloyed) interface (RI) structure is also studied and the ITR of such interfaces is increased by 5 times. By decomposing the heat flux, ${ }^{25-27}$ the underlying mechanism is explained clearly. The result of the frequency dependent transmission coefficient shows that the ability of effective phonons to pass through the interface is: mass graded interface $>$ perfectly abrupt interface $>$ rough interface. At the same time, the mechanisms of how the high frequency phonons evolve in the interfacial region are found to be distinct for these three types of interfaces. In addition, with the help of a phonon wave packet method, ${ }^{28}$ the underlying mechanism for the frequency dependent transmission coefficient changing with different interfacial structures is interpreted in detail.
Our large-scale nonequilibrium molecular dynamics (NEMD) simulations, performed with LAMMPS package ${ }^{29}$ and the standard 12-6 Lennard-Jones (LJ) potential, considered Ar-heavy $\mathrm{Ar}$ crystalline lattice systems with the lattice constant of $0.529 \mathrm{~nm}$. The ratio between heavy Ar and regular Ar is 10. Three different types of interfaces are studied: the perfectly abrupt interface, rough alloyed interfaces with different thicknesses (1.1-63.5 nm) and the volume concentration of heavy $\operatorname{Ar}$ (0.13-0.90), and mass graded interfaces with thicknesses ranging from 4.23 to $423 \mathrm{~nm}$. In our simulations, we construct the mass graded layer by gradually increasing the mass in the interface region from the regular argon to the heavy argon. Then, there are two parameters we can control in the mass graded layer: the number of added mass and the thickness of each layer. In our simulations, the number of mass ranges from 8 to 100 and the thickness of each layer ranges from $0.529 \mathrm{~nm}$ to $4.232 \mathrm{~nm}$. Detailed structures of the interfaces are shown in Fig. 1(a). The cross-sectional area of all models in our simulations is $4.232 \times 4.232 \mathrm{~nm}^{2}$. Periodic boundary conditions are applied in lateral directions, while non-periodic boundary conditions are implemented in the heat flux direction, which is perpendicular to the interfaces. To generate steady heat current flowing through the systems, the atoms located at the distance $L_{\text {bath }}$ from the left and right ends of the system are coupled to hot and cold Nose-Hoover thermostats at temperatures $T+\Delta T / 2$ and $T-\Delta T / 2$, respectively. Two left and right-most layers of atoms are maintained at fixed positions to prevent large deformations and translational movements of the systems. The mean temperature of the entire system is fixed at $T=10 \mathrm{~K}$ and the temperature difference between the two thermostats is $\Delta T=5 \mathrm{~K}$. It has already been proved in a previous study that the non-linear effect caused by the temperature difference of $5 \mathrm{~K}$ in the Ar system with the system temperature of $10 \mathrm{~K}$ can be ignored. ${ }^{26}$ We perform $8.4 \times 10^{6}$ NEMD steps with a time step of $5 \mathrm{fs}$, corresponding to the total running time of $42 \mathrm{~ns}$. In all of our simulations, the first $20 \mathrm{~ns}$ is used to obtain the steady (a)

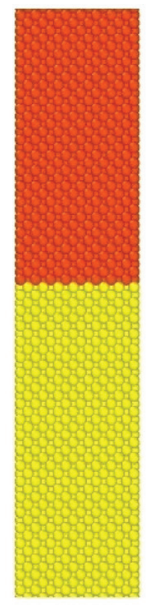

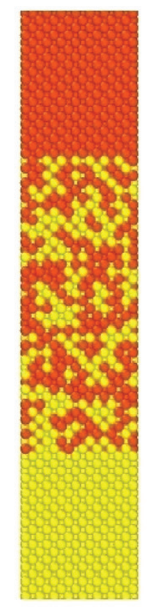

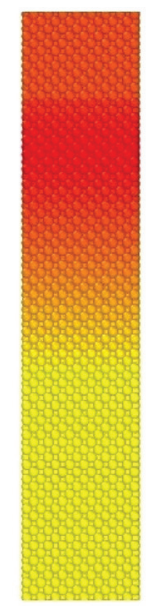

(b)
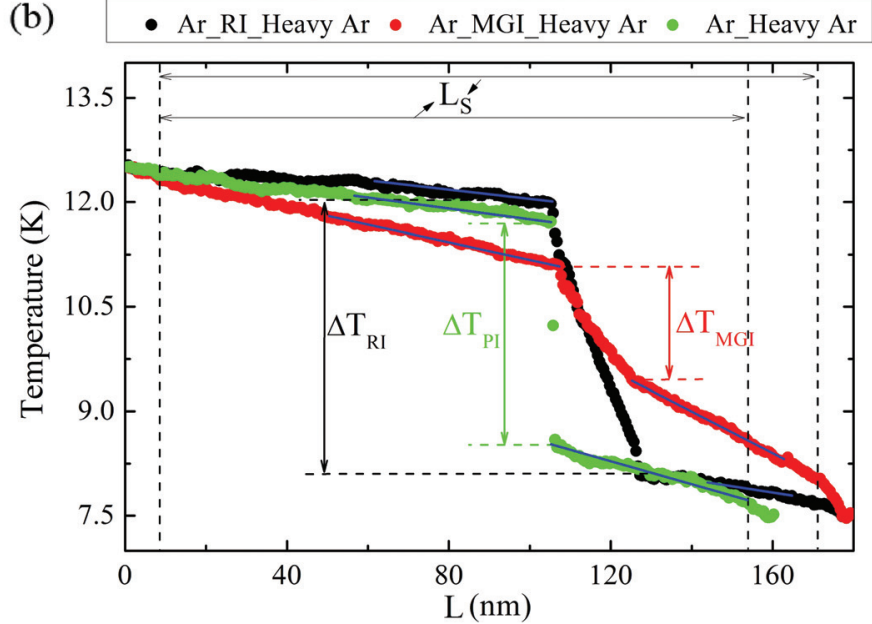

Fig. 1 (a) Side view of three interface structures studied in this work. From left to right: perfectly abrupt interface, rough interface, mass graded interface. (b) Steady state temperature profile for the three kinds of interface structures shown in (a) calculated by nonequilibrium molecular dynamics simulations with average system temperature $T=10 \mathrm{~K}$. 
temperature gradient and heat flux, and then the following $20 \mathrm{~ns}$ is used to calculate the average temperature gradient and heat flux, which are used to compute the interfacial thermal resistance. We have verified that the system temperature, the total energy of the system and the heat flux reach a steady state after running 20 ns. A typical averaged temperature profile obtained from the NEMD simulation is shown in Fig. 1b. The temperature drop $\Delta T$ at the interface is estimated from the difference between the linear temperature profiles extrapolated to the interface as illustrated in Fig. 1 (b), where $\Delta T_{\mathrm{PI}}, \Delta T_{\mathrm{RI}}$ and $\Delta T_{\mathrm{MGI}}$ represent the temperature drop at the perfectly abrupt interface, rough interface, and mass graded interface, respectively. Then, the ITR can be obtained by $R_{\text {int }}=A \Delta T / q$ and $G=$ $1 / R_{\text {int }}$, where $A$ and $q$ are the cross-sectional area and heat current, and $G$ is the overall interfacial thermal conductance. It is well known that ITR depends on the length of the two materials in contact, which originates from the truncation of long MFP phonons at a finite length. In order to guarantee that all possible phonons can appear in our models, the length of $\mathrm{Ar}$ and heavy Ar parts should be long enough to accommodate phonons with the largest MFP. Here, using the time domain normal mode analysis (TDNMA) ${ }^{30,31}$ with $8 \times 8 \times 8$ unit cell systems, we obtain the largest MFPs of Ar and heavy Ar to be $65 \mathrm{~nm}$ and $24 \mathrm{~nm}$, respectively. Therefore, in all our models we choose the length of the Ar lead as $100 \mathrm{~nm}$ and the heavy Ar lead as $50 \mathrm{~nm}$. In this way, the ITR can be regarded as independent of the length of two leads. Meanwhile, we also calculate the ITR of the abrupt interface system with different lengths and crosssectional areas. The results (not shown for brevity) demonstrate that the size effects in our models can be ignored.

The ITR results obtained for the three interfaces are summarized in Fig. 2. We first notice that almost all rough interfaces have a larger ITR with respect to the perfectly abrupt interface case. It is quite understandable and well documented that making the interface rough is a quite efficient way to increase the ITR. ${ }^{8,10,16}$ Here, our results show that the value of ITR can be enhanced by as large as about 5 times when the alloyed material is used as an additive layer. Such a huge improvement of ITR implies that the method of making the interface rough has immense potential in the field of thermal insulation, but not beneficial for heat dissipation in electronic cooling. Such interfacial structures have already been synthesized in experiments. ${ }^{8}$ Using atomistic Green's function method, Li et $a l .{ }^{32}$ and Gu et $a .^{33}$ also found that adding a disordered layer in the interface region can enhance the ITR dramatically. Moreover, both Li's work ${ }^{32}$ and our paper (Fig. 3) revealed that the high frequency phonons scatter quite strongly. It is also worth noting that, when the thickness of the rough additive layer decreases to a few atomic layers, the ITR can even surpass that for the perfectly abrupt interface. However, based on our current result and others' work, such a way to reduce the ITR is not efficient, since the largest reduction percentage achieved so far is only in the range of $20 \%-50 \%,{ }^{15,16}$ i.e. by up to 2 folds. In addition, manufacturing a structure with only a few atomic layers is not an easy task, meaning this method is impractical in realistic applications.

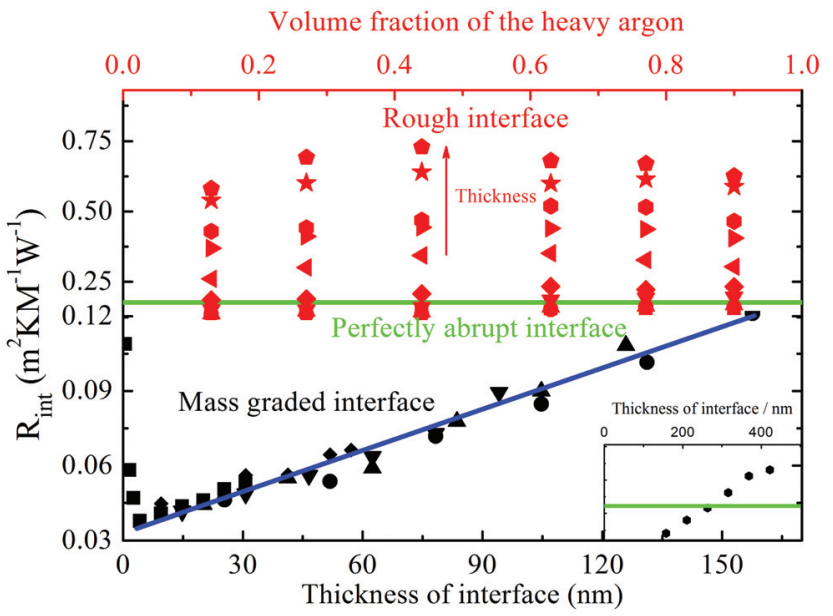

Fig. 2 The overall interfacial thermal resistance across the entire interface region calculated by Fourier's law. The black (red) symbols represent the results of the mass graded (rough) interface with various thicknesses (volume fraction). The arrow indicates the direction of increasing thickness. The green line is the interfacial thermal resistance of the perfectly abrupt interface. The blue line is a fitting line. (Inset) The ITR of the mass graded interface goes beyond that for the perfectly abrupt interface, when the mass graded interface is thick enough (at least $300 \mathrm{~nm}$ ). For the rough interface, the different symbols represent different thicknesses of the interface layer. For the mass graded interface, the different symbols mean a different number of atom mass added in the mass graded layer.

Surprisingly, for mass graded additive layers the ITR can be suppressed by as large as about 6 fold as compared with that for the perfectly abrupt interface. Such finding shows that the mass graded structures have great promise in thermal management of electronic cooling. It is also interesting to find that the ITR linearly increases with thickness. Actually, the ITR of such a structure is even larger than that of the perfectly abrupt interface when the thickness is large enough (the inset of Fig. 2). It is not surprising to find this phenomenon because, as the thickness increases the thermal resistance induced by the mass graded layer itself increases as well and eventually dominates the ITR across the entire interface region. What is more, the ITR of the mass graded interface approaches the ITR of the abrupt interface, when the additive layer is thin enough, e.g. the ITR of the mass graded interface with the smallest possible thickness of $0.529 \mathrm{~nm}$ ( 1 unit cell of Ar lattice) reaches $62 \%$ of that for the abrupt interface (Fig. 2).

Now we analyze the underlying mechanism of thermal transport across different interfacial additive layers by analyzing the phonon process in NEMD simulations. The expression for the heat current $q_{i j}$ between atoms $i$ and $j$ can be given by ${ }^{26,27}$

$$
q_{i j}=\frac{1}{2}\left\langle F_{i j}\left(v_{i}+v_{j}\right)\right\rangle,
$$

where $F_{i j}$ is the force between two atoms and $v$ is the velocity of atoms, \langle\rangle denotes the steady-state nonequilibrium ensemble average. Meanwhile, it has been proved that the spectral decomposition of heat current is related to the correlation 
(a)

\section{(a)}
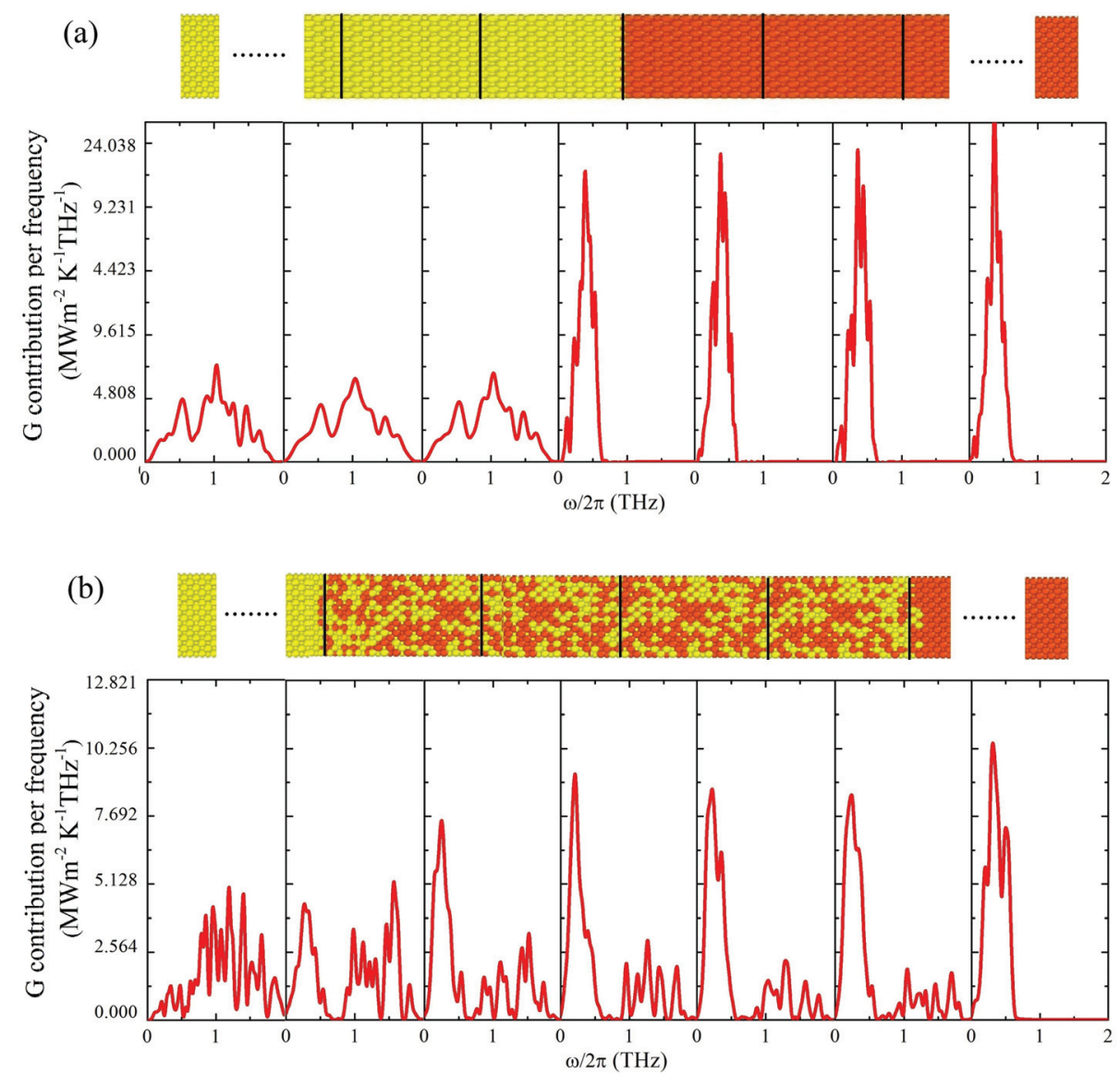

(c)
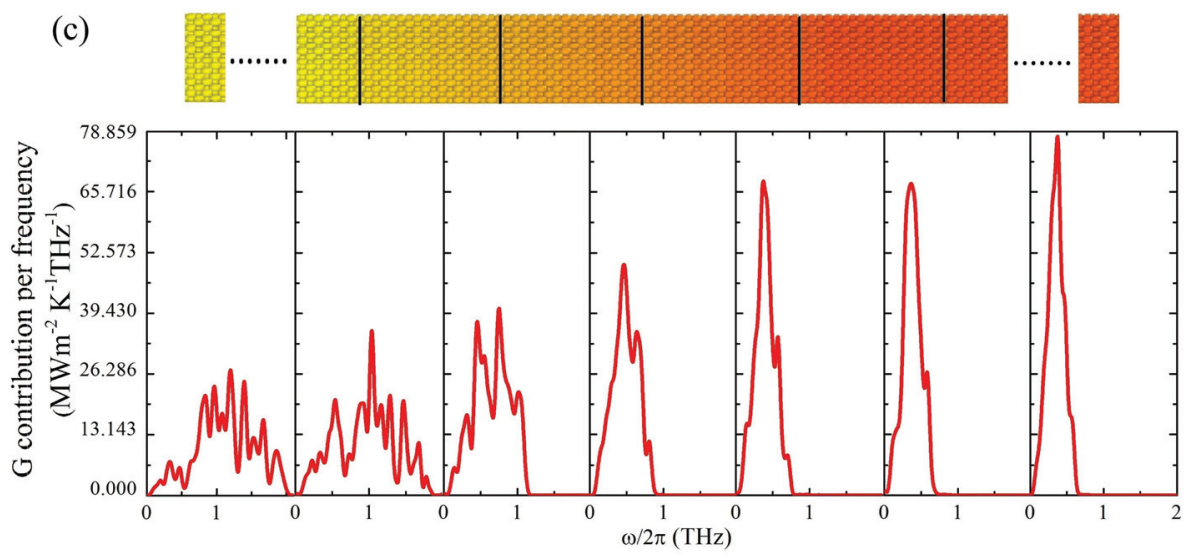

Fig. 3 Frequency dependent interfacial thermal conductance (FDITC) for (a) the perfectly abrupt interface, (b) rough interface, and (c) mass graded interface. The first and last panels in (a) to (c) represent the FDITC in the middle of the left and right leads, respectively. The second to fifth panel is the FDITC at the positions indicated by the black lines in the corresponding structure models. The temperature at the interface is used to obtain the FDITC result.

time between force and velocity. ${ }^{25,26,34}$ Therefore, $q_{i j}$ can be further given in the expression below after mathematical operations (details can be found in ref. 26 and 27)

$$
q_{i j}=\frac{1}{2} \int_{-\infty}^{+\infty} \int_{-\infty}^{+\infty} C_{i j}(\tau) e^{i \omega \tau} \mathrm{d} \tau \frac{\mathrm{d} \omega}{2 \pi} .
$$

where $C_{i j}$ is the auxiliary correlation term with the expression of $C_{i j}(\tau)=\left\langle F_{i j}(\tau) \cdot\left(v_{i}(0)+v_{j}(0)\right)\right\rangle$. In addition, it is usual to assume that $F_{i j}^{\alpha} \approx \sum_{\beta} u_{i}^{\beta} K_{i j}^{\alpha \beta}$ in the low temperature system, where $K_{i j}^{\alpha \beta}$ is the second order interatomic force constants. Then, eqn (2) can be rewritten as (details can be found in ref. 25, 35)

$$
q_{i j}=\int_{0}^{+\infty}\left(-\frac{2}{t_{\operatorname{simu}} \omega} \sum_{\alpha, \beta \in\{x, y, z\}} \operatorname{Im}\left\langle\tilde{v}_{i}^{\alpha}(\omega)^{*} K_{i j}^{\alpha \beta} \tilde{v}_{j}^{\beta}(\omega)\right\rangle\right) \frac{\mathrm{d} \omega}{2 \pi} .
$$


Finally, we can write the frequency dependent thermal conductance across an individual interface as

$$
G(\omega)=-\frac{2}{t_{\mathrm{simu}} \omega A \Delta T} \sum_{i \in \mathrm{L}, j \in \mathrm{R}} \sum_{\alpha, \beta \in\{x, y, z\}} \operatorname{Im}\left\langle\tilde{v}_{i}^{\alpha}(\omega)^{*} K_{i j}^{\alpha \beta} \tilde{v}_{j}^{\beta}(\omega)\right\rangle
$$

where $t_{\text {simu }}$ is the simulation time, " $\mathrm{L}$ " and " $\mathrm{R}$ " means the left and right region separated by the interface. In order to compare our results consistently, all the temperature drops used in eqn (4) are the interfacial temperature drops.

Using eqn (4), we can obtain the frequency dependent thermal conductance as illustrated in Fig. 3. We notice that the first panel of all three systems is not exactly the same, even if they have the same crystal structure. In our three models, the temperature gradient in the regular Ar part is different (Fig. 1) despite the same crystal structure. However, we can easily find that the shape of these three pictures is similar except for the different noise in it. Such noise is inevitable when one uses eqn (4) to obtain the frequency dependent heat flux. It seems that the only method to reduce the noise is to enlarge the temperature gradient in the system, which will certainly increase the non-linear effect in the system as well. Therefore, there is somehow a compromise between the small noise in the frequency dependent heat flux and the large nonlinear effect from the thermostats. On the one hand, we know that for any kind of interfaces only phonons with frequency below the cutoff frequency of the second material, i.e. $\omega_{\mathrm{c}}^{\text {heavy argon }}=$ $0.68 \mathrm{THz}$, can carry heat across the interface, because higher frequency modes cannot propagate in the heavy Ar, which is determined by its lowered phonon dispersion curves (inset of Fig. 5). On the other hand, for low temperature systems, it is widely regarded that the transmission coefficient can be written as $\Gamma(\omega)=G(\omega) / k_{\mathrm{b}},{ }^{26,36}$ which means we can simply regard the $G$ as $\Gamma$. Then, it is easy to observe that the transmission coefficient of phonons that can pass through the rough interface has a lower value than that for the perfectly abrupt interface (comparing Fig. 3(a) and (b)). While for the mass graded interface, the transmission coefficient of phonons is substantially higher than that for the perfectly abrupt interface (comparing Fig. 3(a) and (c)). However, when the size of the rough interface decreases to several atomic layers, the transmission coefficient of phonons has a larger value than that for the perfectly abrupt interface (results not shown for brevity). Consequently, it results in the incremental drop in the ITR (about $20 \%-40 \%$ in our work, see Fig. 2).

We now turn to the mechanism of how the phonons with frequency larger than the cutoff frequency of heavy Ar evolve in the interface region. For the perfectly abrupt interface (Fig. 3(a)), phonons with frequency below the cutoff frequency have the possibility to pass through the interface. All the phonons with frequency beyond cutoff frequency are reflected by the interface. When the interface becomes rough, high frequency phonons $\left(\omega>\omega_{\mathrm{c}}^{\text {heavy argon }}\right)$ are presented in the interface region (see the $2^{\text {nd }}$ to $5^{\text {th }}$ pictures in Fig. 3(b)), due to the possibility of the population of full frequency range phonons for the alloyed interface. We also observe that the heat energy carried by these high frequency phonons decreases gradually from left to right and almost annihilates at the right interface between the alloyed layer and the heavy $\mathrm{Ar}$ (the $5^{\text {th }}$ picture in Fig. 3(b)). Thus, we can conclude that for sandwiched rough layers, the main mechanism for the disappearance of the high frequency phonons in the right lead is phonon scattering in the course of traveling. However, it is worth pointing out that when the thickness of the rough interface decreases to a few atomic layers, the dominant mechanism is the phonon reflection at the interface, since there is no enough space for such phonons to scatter. Therefore, for the rough interface there are two different regimes of interfacial heat transfer, which originates from the competition between phonon scattering and the thickness of the rough interface. When a mass graded material is added into the interface region (Fig. 3(c)), phonons with a frequency higher than $0.68 \mathrm{THz}$ are truncated gradually, due to the gradual increase in the transmission coefficient with the increasing number of layers (each layer and its adjacent layer with slight mass difference can be regarded as an interface), as we will see later. Thereby, we see from Fig. 3(c) that the peak of the frequency dependent thermal conductance around 0.38 $\mathrm{THz}$ becomes sharper as phonons travel across the mass graded region, meaning that the majority of thermal energy is transmitted by these medium frequency phonons. This can be understood in terms of their relatively high transmission coefficient (as we will see later in Fig. 5) and decent phonon group velocities as compared with low frequency phonons.

In order to gain more insight into the mechanism of phonon transport across these three typical interfaces, we perform the wave packet dynamics simulation ${ }^{37,38}$ to compute the frequency dependent transmission coefficient. This method has been successfully used to analyze phonon transport across various interfaces. ${ }^{37}$ The phonon wave packets are generated from linear combinations of vibrational eigenstates of bulk Ar, which are obtained from harmonic lattice dynamics calculations using PHONOPY software. ${ }^{39}$ To generate a wave packet centered in a wave vector space at $k_{0}$ and in a geometrical space at $z_{0}$, we displace the atoms according to ${ }^{37}$

$$
u_{i l \mu}=A_{\mathrm{m}} \varepsilon_{i \mu \lambda}\left(k_{0}\right) \exp \left[i k_{0}\left(z_{1}-z_{0}\right)\right] \exp \left[-\eta^{2}\left(z_{1}-z_{0}\right)^{2}\right]
$$

where $z_{l}$ is the position of the $l^{\text {th }}$ unit cell, $u_{i l \mu}$ is the $\mu^{\text {th }}$ component of displacement for the atom $i$ in the $l^{\text {th }}$ unit cell, $A_{\mathrm{m}}$ is the amplitude of the wave, $\varepsilon_{i \mu \lambda}\left(k_{0}\right)$ is the eigenvector for the $\lambda^{\text {th }}$ phonon branch at $k_{0}$. The value of $\eta$ is selected to keep the wave packet extent to be about 100 unit cells. The initial atomic velocities are set according to the formula given in ref. 28.

Here, we choose one typical longitudinal acoustic phonon in the medium frequency region $(\omega / 2 \pi=0.23 \mathrm{THz})$ among all effective phonons as an example. For the perfectly abrupt interface, most of the incoming energy carried by the wave packet can transfer across the interface and the remaining part is reflected by the interface directly (Fig. 4(a)). In contrast, for the structure with a rough interface, the majority of incident energy is quite difficult to go through the interface region, due 
(a)
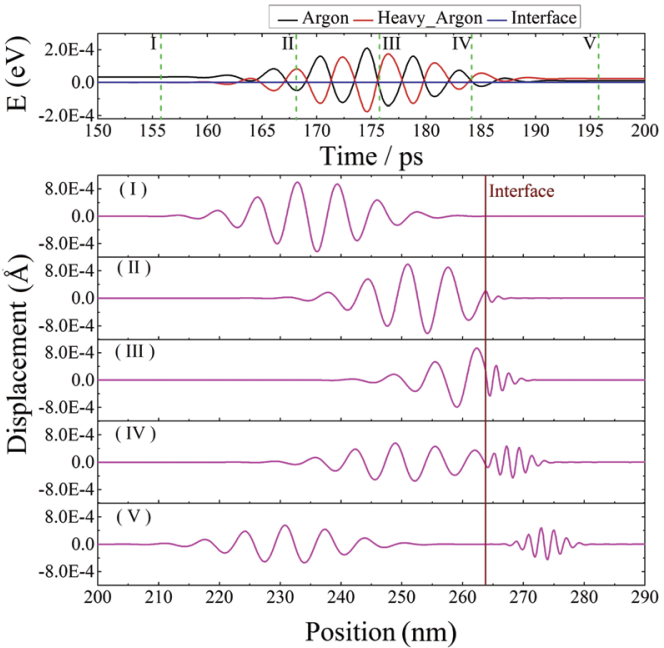

(b)
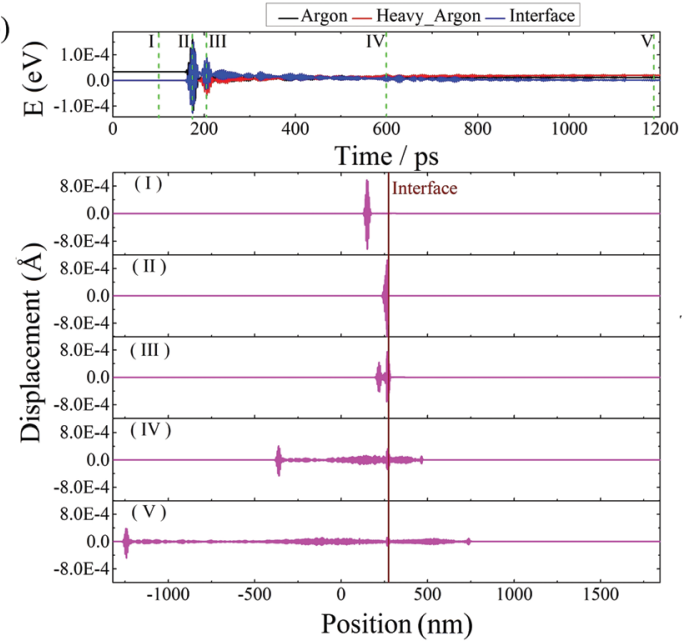

(c)
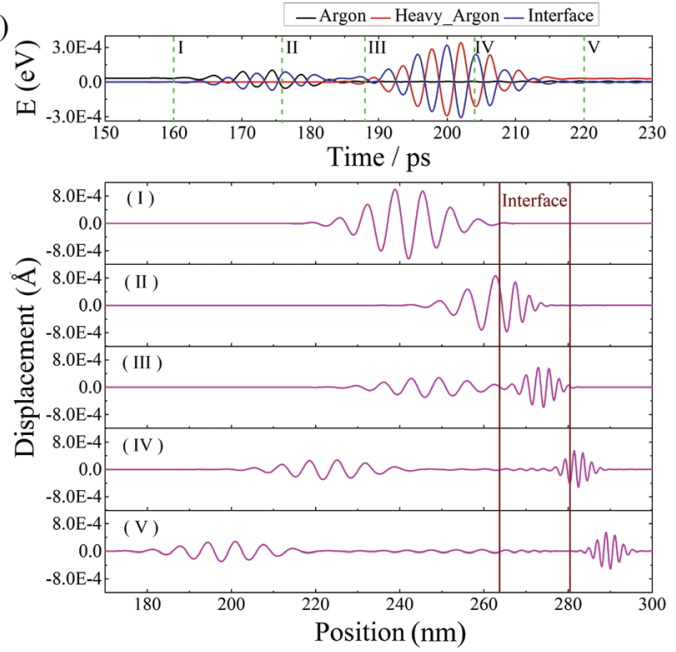

Fig. 4 (Top) Phonon energy evolvement as a function of time by wave packet simulation and (Bottom) snapshots of displacements for a longitudinal acoustic wave packet with a frequency of $0.23 \mathrm{THz}$ at some typical timesteps indicated by the Roman numerals for (a) the perfectly abrupt interface, (b) rough interface, and (c) mass graded interface. Color coding: black, argon; red, heavy argon; blue, interface. The brown vertical lines in the bottom panel indicate the position of interfaces. to the strong scattering between atoms with different masses in this region (Fig. 4(b)). Only a small portion of phonon's energy can transport across the interface, resulting in a much lower transmission coefficient than that of the perfectly abrupt interface. In the case of the mass graded interface, the major energy reflection (but note that the transmission coefficient is rather high) occurs at the first interface position (Fig. 4(c)), i.e. when the phonon traveling in the regular Ar lead suddenly encounters the mass graded structure. After that, very little phonon energy is reflected as the phonon continues to travel across the mass graded region, although each of the two adjacent mass graded layers can be regarded as an interface. This is mainly because the acoustic impedance at each mass graded interface decreases with the increasing layer number. The details are explained below.

In Fig. 5 we compare the frequency dependent transmission coefficient for the three types of interfaces with two typical interface thicknesses. Due to the fact that the long MFP phonons (with respect to the thickness of interface) can pass the thin layer with small interference, the transmission coefficient of these phonons is relatively higher than that for high frequency phonons (see the low frequency region in Fig. 5). As can be seen from Fig. 5, for the rough interface, with the increasing interface thickness, the frequency dependent transmission coefficient has a downward and leftward shift, indicating that the number of phonons with a lower transmission coefficient than that of the perfectly abrupt interface increases, which leads to an increased ITR (Fig. 2 and 3(b)). When the thickness of the rough interface region is reduced to several atomic layers (8 unit cells, left panel of Fig. 5), the transmission coefficient of almost all phonons below the cutoff frequency increases as compared with that for the thicker rough interface, especially for the medium frequency range (0.15-0.58 THz), leading to the ITR even lower than that of the perfectly abrupt interface (Fig. 2). For the mass graded interface we find that almost all the effective phonons have substantially high transmission coefficient (between 0.85 and 1) as compared to both the perfectly abrupt interface and rough interface. The transmission coefficient sharply drops down to zero as the phonon frequency approaches the cutoff frequency (0.51 and $0.68 \mathrm{THz}$ for longitudinal acoustic and transverse acoustic phonon, respectively). As a result, the ITR of the mass graded interface decreases largely (by 6 fold at the most as shown in Fig. 2 and 3(c)).

Before closing, we use the AMM model to predict the transmission coefficient of the perfectly abrupt interface and the mass graded interface. For the perfectly abrupt interface, the AMM formula says ${ }^{14}$

$$
\alpha_{1 \rightarrow 2}=\frac{4 Z_{1} Z_{2}}{\left(Z_{1}+Z_{2}\right)^{2}},
$$

where $Z_{i}=\rho_{i} c_{i}$ is the acoustic impedance of the regular Ar and heavy Ar leads, which is equal to the product of mass density and sound velocity. In the case of the same interatomic interaction strength, the acoustic impedance is proportional to the 


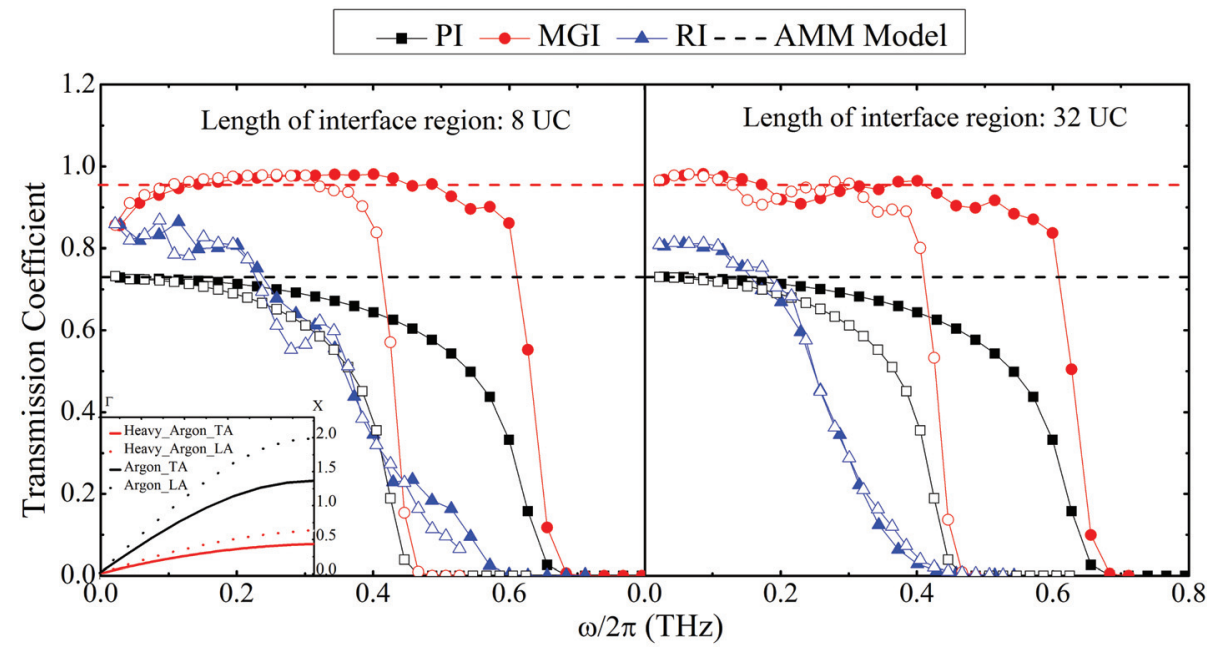

Fig. 5 Phonon frequency dependent transmission coefficients for the perfectly abrupt interface (black symbols), mass graded interface (red symbols) and rough interface (blue symbols) with an interface thickness of (left) 8 unit cells and (right) 32 unit cells. The black and red dashed lines denote the transmission coefficient at a long-wavelength limit using the acoustic mismatch model for the perfectly abrupt interface and mass graded interface, respectively. The filled and open symbols denote the longitude acoustic (LA) and transverse acoustic (TA) branches, respectively. (Inset) Phonon dispersion curves of regular Ar (black lines) and heavy Ar (red lines).

square root of atomic mass. Then we predict the AMM transmission coefficient of the perfectly abrupt interface to be 0.73 for $\omega \rightarrow 0$, which is consistent with the wave packet results shown in Fig. 5. For the mass graded interface, we first calculated the transmission coefficient of each interface composed of two adjacent mass graded structures using the AMM model. We found that these transmission coefficient values are very close to 1 , due to extremely low mass mismatch in the mass graded interface structure. Then we calculated the overall transmission coefficient according to the following equations $^{28}$

$$
\begin{gathered}
\alpha_{1 \rightarrow 3}=\alpha_{1 \rightarrow 2} \alpha_{2 \rightarrow 3} \sum_{m=1}^{\infty}\left(1-\alpha_{1 \rightarrow 2}\right)^{m}\left(1-\alpha_{2 \rightarrow 3}\right)^{m}, \\
\alpha_{1 \rightarrow N+1}=\alpha_{1 \rightarrow N} \alpha_{N \rightarrow N+1} \sum_{m=1}^{\infty}\left(1-\alpha_{1 \rightarrow N}\right)^{m}\left(1-\alpha_{N \rightarrow N+1}\right)^{m} .
\end{gathered}
$$

In this way, the final overall transmission coefficient across the entire mass graded interface is calculated to be 0.948 , which is again consistent with the wave packet results shown in Fig. 5 and this value is much higher than that of the perfectly abrupt interface (0.73). Also, we calculated the transmission coefficient of the directly transmitted wave, i.e. only counting the phonon energy that transmits across each mass graded interface with a gradually increasing transmission coefficient, and neglecting the energy that goes back-and-forth across the multi-layers, by

$$
\alpha_{1 \rightarrow N+1}=\prod_{n=1}^{N} \alpha_{n \rightarrow n+1} .
$$

The obtained directly transmitted transmission coefficient is 0.947 , which is very close to the above overall transmission coefficient of the mass graded interface, indicating that the enhanced transmission coefficient of the mass graded interface compared to that of the perfectly abrupt interface is purely due to the reduced mass mismatch and the phonon process in the mass graded interface region is dominated by the simple direct transmission of phonons without observable reflection and go-back-and-forth inside the mass graded interface region. Actually, this can be clearly seen from the wave packet results as shown in Fig. 4(c). Note that the AMM model reproduces the transmission coefficient of the low frequency phonons but does not agree very well with the high frequency phonons (Fig. 5), due to the fact that the AMM model is based on the acoustic impedance and only valid for the long-wavelength limit. The results of both the decomposed interfacial thermal conductance and transmission coefficient computed using the wave packet method show that the main reason for the reduction of ITR for the mass graded interface is the enhancement of the transmission coefficient of phonons, which comes from the small mass mismatch between two neighboring layers in the mass graded interface region (Fig. 3 and 5).

To summarize, using a model structure of regular Ar and heavy $\mathrm{Ar}$ in contact, we have presented the superiority of largely decreasing the interfacial thermal resistance by sandwiching a mass graded layer. While the widely adopted alloyed interface usually increases the overall interfacial thermal resistance by several times as compared with the perfectly abrupt interface, except for atomically thin layers, the mass graded structure substantially reduces the overall resistance by 6 fold. Using the atomic heat flux spectrum theory, the governing mechanisms are well explained. There are two different regimes of interfacial heat transfer across the rough interface, which originates from the competition between phonon 
scattering and the interface thickness. When the thickness is large enough, there is enough space for high frequency phonons to scatter and then the interfacial thermal resistance will increase, while when the thickness is reduced to only a few atomic layers, there is no enough space for phonon scattering and thus the interfacial thermal resistance decreases to some extent. Moreover, the detailed phonon process for high frequency phonons across three types of interfaces $(\omega>$ $\omega_{\mathrm{c}}^{\text {heavy argon }}$ ) are studied using the wave packet method and the acoustic mismatch model. For the perfectly abrupt interface, all the phonons with a frequency above the cutoff frequency of heavy Ar are reflected and for the alloyed interface, the high frequency phonons gradually disappear through phonon scattering in the alloyed region. In contrast, for the mass graded interface the minor phonon reflection occurs when the phonons first reach the mass graded area. After that, the phonons can easily transmit across the subsequent multilayers with gradually increasing transmission coefficient and hardly reflect. Consequently the major thermal energy transport turns out to be carried by medium frequency phonons. Wave packet simulation also show that, for the rough interface the short and medium mean free path (corresponding to medium and high frequency) phonons have a lower transmission coefficient with respect to those in the perfectly abrupt interface due to the strong phonon scattering, while the long mean free path (corresponding to low frequency) phonons have the opposite trend, since they can transport across the thin layer with small interference. For the mass graded interface, almost all the phonons with a frequency below the cutoff frequency of heavy Ar have significantly higher transmission coefficients, primarily due to the small mass mismatch between the adjacent layers in the interface region, and therefore, the interfacial thermal resistance is dramatically reduced. Although the present results are based on simple lattice models, our findings shed light on the field of interfacial heat transfer in terms of improving the heat dissipation efficiency in electronic cooling. We truly believe that our model structures can be realized in nanoscale systems experimentally. ${ }^{24,40,41}$ To support our view, we use a realistic model (Ge-heavy Ge), which can be fabricated in experiments using a similar method presented in ref. 42. The popular Tersoff potential $^{43}$ is used for the Ge-Ge interactions. The crosssection and length of all Ge-heavy Ge models are $3.4 \times$ $3.4 \mathrm{~nm}^{2}$ and $56.7 \mathrm{~nm}$, respectively. The same NEMD method is applied to the Ge-heavy Ge systems. The temperature of the entire system is fixed at $T=300 \mathrm{~K}$ and the temperature difference between the two thermostats (heat source and heat sink) is $\Delta T=50 \mathrm{~K}$ during the NEMD run. The total simulation time of NEMD is $42 \mathrm{~ns}$ with a time step of $4 \mathrm{fs}$. For the mass graded interfaces, the overall thermal conductance is found to be as large as about 2 times with respect to the abrupt interface model (Fig. 6). It is worth noting that the anharmonicity in our system can be ignored when the temperature is low enough, which is also proved by Sääskilahti et al. ${ }^{26,44}$ Furthermore, both other researchers ${ }^{8}$ and we (Fig. 6) prove that, even at room temperature in which the phonon anharmonicity is

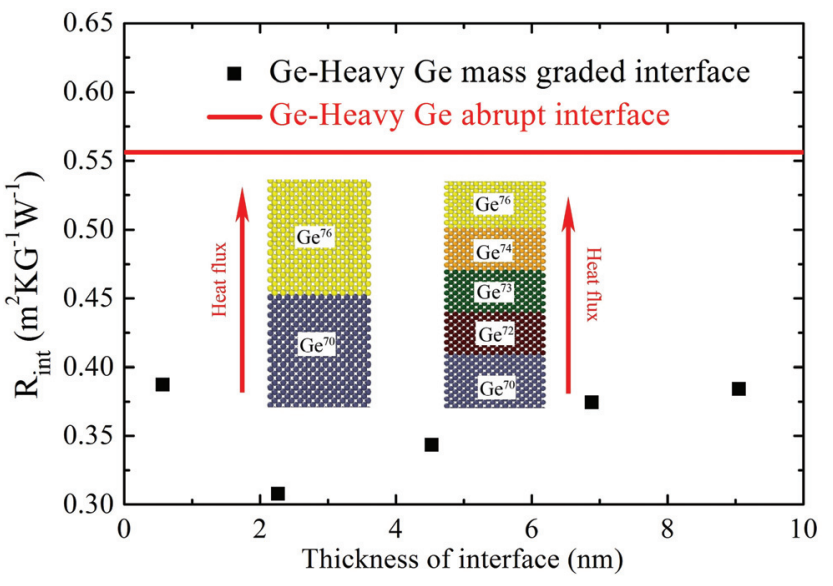

Fig. 6 The overall interfacial thermal resistance across the entire Geheavy Ge mass graded region as a function of the interface thickness. The red horizontal line represents the thermal resistance for the abrupt Ge-heavy Ge interface.

important, the interfacial thermal resistance can still be reduced or enhanced largely with structure optimization of the interface. Therefore, our main conclusions in the manuscript still hold at least around the room temperature, where the anharmonicity is relatively strong.

\section{Acknowledgements}

Simulations were performed with computing resources granted by the Jülich Aachen Research Alliance-High Performance Computing (JARA-HPC) from RWTH Aachen University under Project No. jara0127.

\section{References}

1 P. K. Schelling, S. R. Phillpot and P. Keblinski, J. Appl. Phys., 2004, 95, 6082.

2 G. Chen, Phys. Rev. B: Condens. Matter, 1998, 57, 14958.

3 R. S. Prasher, X. J. Hu, Y. Chalopin, N. Mingo, K. Lofgreen, S. Volz, F. Cleri and P. Keblinski, Phys. Rev. Lett., 2009, 102, 105901.

4 D. G. Cahill, P. V. Braun, G. Chen, D. R. Clarke, S. Fan, K. E. Goodson, P. Keblinski, W. P. King, G. D. Mahan and A. Majumdar, Appl. Phys. Rev., 2014, 1, 011305.

5 P. L. Kapitza, J. Phys., 1941, 4, 181.

6 M. D. Losego, M. E. Grady, N. R. Sottos, D. G. Cahill and P. V. Braun, Nat. Mater., 2012, 11, 502.

7 P. J. O. Brien, S. Shenogin, J. Liu, P. K. Chow, D. Laurencin, P. H. Mutin, M. Yamaguchi, P. Keblinski and G. Ramanath, Nat. Mater., 2013, 12, 118.

8 P. E. Hopkins, J. C. Duda, C. W. Petz and J. A. Floro, Phys. Rev. B: Condens. Matter, 2011, 84, 035438. 
9 P. E. Hopkins, M. Baraket, E. V. Barnat, T. E. Beechem, S. P. Kearney, J. C. Duda, J. T. Robinson and S. G. Walton, Nano Lett., 2012, 12, 590.

10 L. M. Phinney, J. R. Serrano, T. E. Beechem and P. E. Hopkins, Phys. Rev. B: Condens. Matter, 2010, 82, 085307.

11 P. E. Hopkins, J. C. Duda, S. P. Clark, C. P. Hains, T. J. Rotter, L. M. Phinney and G. Balakrishnan, Appl. Phys. Lett., 2011, 98, 161913.

12 S. Merabia and K. Termentzidis, Phys. Rev. B: Condens. Matter, 2014, 89, 054309.

13 Z. Liang, K. Sasikumar and P. Keblinski, Phys. Rev. Lett., 2014, 113, 065901.

14 E. T. Swartz and R. O. Pohl, Rev. Mod. Phys., 1989, 61, 605.

15 Z. Tian, K. Esfarjani and G. Chen, Phys. Rev. B: Condens. Matter, 2012, 86, 235304.

16 T. S. English, J. C. Duda, J. L. Smoyer, D. A. Jordan, P. M. Norris and L. V. Zhigilei, Phys. Rev. B: Condens. Matter, 2012, 85, 035438.

17 X. W. Zhou, R. E. Jones, C. J. Kimmer, J. C. Duda and P. E. Hopkins, Phys. Rev. B: Condens. Matter, 2013, 87, 094303.

18 C. Shao and H. Bao, Int. J. Heat Mass Transfer, 2015, 85, 33.

19 E. S. Landry and A. McGaughey, J. Appl. Phys., 2010, 107, 013521.

20 S. Lu and A. J. McGaughey, AIP Adv., 2015, 5, 053205.

$21 \mathrm{M}$. Hu and D. Poulikakos, Int. J. Heat Mass Transfer, 2013, 62, 205.

22 M. Hu, X. Zhang, D. Poulikakos and C. P. Grigoropoulos, Int. J. Heat Mass Transfer, 2011, 54, 5183.

$23 \mathrm{M}$. Hu, J. V. Goicochea, B. Michel and D. Poulikakos, Nano Lett., 2009, 10, 279.

24 C. L. Wu, W. C. Hsu, H. M. Shieh and W. C. Liu, Appl. Phys. Lett., 1994, 64, 3027.

25 Y. Chalopin and S. Volz, Appl. Phys. Lett., 2013, 103, 051602.
26 K. Sääskilahti, J. Oksanen, J. Tulkki and S. Volz, Phys. Rev. B: Condens. Matter, 2014, 90, 134312.

27 Y. Zhou and M. Hu, Phys. Rev. B: Condens. Matter, 2015, 92, 195205.

28 P. K. Schelling and S. R. Phillpot, J. Appl. Phys., 2003, 93, 5377.

29 S. Plimpton, J. Comput. Phys., 1995, 117, 1.

30 Y. Zhou, X. L. Zhang and M. Hu, Phys. Rev. B: Condens. Matter, 2015, 92, 195204.

31 A. J. Ladd, B. Moran and W. G. Hoover, Phys. Rev. B: Condens. Matter, 1986, 34, 5058.

32 X. Li and R. Yang, Phys. Rev. B: Condens. Matter, 2012, 86, 054305.

33 X. Gu, X. Li and R. Yang, Phys. Rev. B: Condens. Matter, 2015, 91, 205313.

34 Y. Chalopin, K. Esfarjani, A. Henry, S. Volz and G. Chen, Phys. Rev. B: Condens. Matter, 2012, 85, 195302.

35 Y. Chalopin, A. Rajabpour, H. Han, Y. Ni and S. Volz, Annu. Rev. Heat Transfer., 2014, 17, 147.

36 K. Sääskilahti, J. Oksanen, S. Volz and J. Tulkki, Phys. Rev. B: Condens. Matter, 2015, 91, 115426.

37 P. K. Schelling, S. R. Phillpot and P. Keblinski, Appl. Phys. Lett., 2002, 80, 2484.

38 S. Sinha, P. K. Schelling, S. R. Phillpot and K. E. Goodson, J. Appl. Phys., 2005, 97, 023702.

39 A. Togo, F. Oba and I. Tanaka, Phys. Rev. B: Condens. Matter, 2008, 78, 134106.

40 J. N. Schulman, J. Vac. Sci. Technol., B, 1983, 1, 644.

41 T. Kawazu and H. Sakaki, Jpn. J. Appl. Phys., 2011, 50, 04DJ06.

42 P. Ferrando-Villalba, A. F. Lopeandía, F. X. Alvarez, B. Paul, C. de Tomás, M. I. Alonso, M. Garriga, A. R. Goñi, J. Santiso and G. Garcia, Nano Res., 2015, 8, 2833.

43 J. Tersoff, Phys. Rev. B: Condens. Matter, 1989, 39, 5566(R).

44 K. Sääskilahti, J. Oksanen, S. Volz and J. Tulkki, Phys. Rev. B: Condens. Matter, 2015, 91, 115426. 